\title{
PERSPECTIVA DE PROFISSIONAIS E ACADÊMICOS DE ENFERMAGEM SOBRE PROMOÇÃO DA SAÚDE
}

\section{PERSPECTIVE ON HEALTH PROMOTION OF PROFESSIONALS AND NURSING ACADEMICS}

\section{PERSPECTIVA DE LOS PROFESIONALES Y ACADÉMICOS DE ENFERMERÍA SOBRE LA PROMOCIÓN DE LA SALUD}

\section{João Pedro Rodrigues Soares ${ }^{1}$, Elen Ferraz Teston ${ }^{2}$, Dandara Novakowski Spigolon ${ }^{3}$, Heloá Costa Borim Christinelli ${ }^{4}$, Maria Antonia Ramos Costa ${ }^{5}$}

Como citar esse artigo: Soares JPR, Teston EF, Spigolon DN, Christinelli HCB, Costa MAR. Perspectiva de profissionais e acadêmicos de enfermagem sobre promoção da saúde. Rev Enferm Atenção Saúde [Internet]. 2021 [acesso em:__]; 10(3):e202134. doi: https://doi.org/ 10.18554/reas.v10i3.4450

\section{RESUMO}

Objetivo: compreender as diferentes perspectivas e práticas de promoção da saúde e prevenção de doenças utilizadas por profissionais da Atenção Primária à Saúde e acadêmicos de enfermagem. Método: pesquisa descritiva, exploratória de abordagem qualitativa, realizada com 11 acadêmicos de enfermagem e 12 profissionais da atenção básica. Os dados foram coletados por meio de entrevistas gravadas e analisados pela análise de conteúdo. Resultados: os entrevistados conceituaram a promoção de saúde de acordo com uma perspectiva ampliada da mesma, embora alguns amalgamem com o conceito de prevenção de doenças. A colaboração da equipe é o principal facilitador para a realização de ações, enquanto a baixa adesão da comunidade é a principal dificuldade. Conclusão: não há consenso entre os profissionais quanto à efetividade das ações desenvolvidas. Os acadêmicos desempenharam um papel fundamental na realização de atividades para a comunidade dentro dos ambientes da atenção básica.

Descritores: Promoção da Saúde; Prevenção de Doenças; Atenção Primária à Saúde; Pessoal de Saúde; Estudantes de Enfermagem.

\footnotetext{
${ }^{1}$ Universidade Estadual de Maringá- UEM. Graduado em Enfermagem pela Universidade Estadual do Paraná UNESPAR, Paranavaí, Paraná, Brasil. Mestrando em Enfermagem pela Universidade Estadual de Maringá, Maringá, Paraná, Brasil.

${ }^{2}$ Universidade Federal do Mato Grosso do Sul - UFMS. Enfermeira. Doutora em Enfermagem. Docente do curso de enfermagem e professora permanente do Programa de Pós-Graduação em enfermagem da Universidade Federal do Mato Grosso do Sul (UFMS), Campo Grande, Mato Grosso do Sul, Brasil.

${ }^{3}$ Universidade Estadual do Paraná - UNESPAR. Enfermeira. Doutora em enfermagem. Docente do colegiado de enfermagem da Universidade Estadual do Paraná (UNESPAR), Paranavaí, Paraná, Brasil.

${ }^{4}$ Universidade Estadual do Paraná - UNESPAR. Bacharel em Enfermagem. Mestre em Tecnologia em Saúde. Docente do colegiado de enfermagem da Universidade Estadual do Paraná (UNESPAR), Paranavaí, Paraná, Brasil.

${ }^{5}$ Universidade Estadual do Paraná -UNESPAR. Enfermeira. Doutora em Enfermagem. Pró-reitora de pesquisa e pós-graduação e docente do colegiado de enfermagem da Universidade Estadual do Paraná (UNESPAR), Paranavaí, Paraná, Brasil.
} 


\begin{abstract}
Objective: to understand how different perspectives and practices of health promotion and disease prevention are used by professionals in Primary Health Care and nursing students. Method: Descriptive, exploratory research with a qualitative approach, carried out with 11 nursing students and 12 primary care professionals. Data were collected through recorded interviews and analyzed by content analysis. Results: interviewed conceptualized health promotion according to its broader perspective, although some crossed with the concept of disease prevention. Team collaboration is the main facilitator for carrying out actions, while low community adherence is the main difficulty. Conclusion: There is no consensus among professionals regarding the effectiveness of the actions developed. Academics play a key role carrying out activities for the community in primary care.
\end{abstract}

Descriptors: Health Promotion; Disease Prevention; Primary Health Care; Health Personnel; Students, Nursing.

\title{
RESÚMEM
}

Objetivo: Conocer las diferentes perspectivas y prácticas de promoción de la salud y prevención de enfermedades utilizadas por los profesionales de Atención Primaria de Salud y estudiantes de enfermería. Método: investigación exploratoria descriptiva con enfoque cualitativo, realizada con 11 estudiantes de enfermería y 12 profesionales de atención primaria. Los datos fueron recolectados a través de entrevistas grabadas y analizados por análisis de contenido. Resultados: entrevistados conceptualizaron la promoción de la salud de acuerdo con una perspectiva más amplia, aunque algunos lo fusionaron con el concepto de prevención de enfermedades. La colaboración del equipo es el principal facilitador para llevar a cabo acciones, mientras que la baja adhesión de la comunidad es la principal dificultad. Conclusión: No existe consenso entre los profesionales sobre la efectividad de las acciones desarrolladas. Los académicos desempeñaron un papel clave en la realización de actividades para la comunidad en atención primaria.

Descriptores: Promoción de la Salud; Prevención de Enfermedades; Atención Primaria de Salud; Personal de Salud; Estudiantes de Enfermería

\section{INTRODUÇÃO}

As mudanças econômicas, políticas, sociais e culturais que ocorrem no mundo desde o século XIX produziram alterações significativas para a vida em sociedade. Ademais, as transições epidemiológica, demográfica e nutricional produziram mudanças expressivas e incluíram novos elementos que determinam o processo saúde-doença. ${ }^{1}$
No que concerne ao conceito ampliado de saúde, desde a criação do Sistema Único de Saúde (SUS), torna-se necessário o desenvolvimento de políticas públicas para promovê-la e garanti-la. Nesse sentido, publicou-se a Política Nacional de Promoção da Saúde (PNPS), que inclui o debate dos condicionantes e determinantes sociais no processo saúdedoença. $^{2}$

A PNPS define as ações de promoção da saúde como um conjunto de 
estratégias e formas de produzir o bemestar biopsicossocial no âmbito individual e coletivo, caracterizando-se pela articulação e cooperação intra e intersetorial, pela formação das Redes de Atenção à Saúde e, a partir disso, estabelecer a criação de meios de comunicação efetivos com as demais redes de proteção social visando à melhoria das condições e modos de viver da população. ${ }^{3}$

As lacunas relacionadas ao desenvolvimento de ações de promoção da saúde e prevenção de doenças são diversas. Dentre os fatores que representam obstáculos na realização dessas ações, evidencia-se a incapacidade de diferenciação dos conceitos supracitados. ${ }^{4}$

Salienta-se que a atuação na promoção da saúde e na prevenção de doenças, dentre outras atividades no escopo da Atenção Básica (AB), constitui uma atribuição comum a todos os integrantes das equipes da Saúde da Família (eSF) e Atenção Básica (eAB), estabelecida na Política Nacional de Atenção Básica (PNAB). ${ }^{5}$

Destaca-se que, dentre as competências e habilidades propostas pelas Diretrizes Nacionais Curriculares do Curso de Enfermagem (DCENF) em 2001, o profissional enfermeiro é responsável pelo desenvolvimento de ações de atenção à saúde e, durante o curso de graduação, deve desenvolver habilidades para realizá- las. ${ }^{6}$ No entanto, estudos apontam dificuldades no desenvolvimento dessas ações. ${ }^{7,8}$

O conhecimento gerado acerca da caracterização das ações educativas que são desenvolvidas na $\mathrm{AB}$ e na formação de novos profissionais da saúde poderá melhorar o desenvolvimento de estratégias educativas ativas que estimulem e incentivem a promoção da qualidade de vida do indivíduo e família. Assim sendo, questiona-se: qual a perspectiva dos acadêmicos e profissionais da $\mathrm{AB}$ em relação ao desenvolvimento das ações de promoção da saúde?

Para responder a esse questionamento, o presente estudo teve como objetivo compreender as diferentes perspectivas e práticas de promoção da saúde e prevenção de doenças utilizadas por profissionais da Atenção Primária à Saúde e acadêmicos de enfermagem.

\section{MÉTODO}

Pesquisa exploratória, de abordagem qualitativa, desenvolvida com profissionais de saúde que atuavam nas Unidades Básicas de Saúde (UBS) e com acadêmicos de Enfermagem de uma instituição pública de ensino superior da região noroeste do Paraná.

O município em que a pesquisa foi realizada contava com 17 UBS, sendo 
cinco destas selecionadas por conveniência para a realização do estudo, resultando em 45 profissionais elegíveis para a coleta de dados. Incluíram-se aqueles que atuavam há mais de seis meses na $\mathrm{AB}$ e excluídos os que estavam de férias, atestado ou licença no período da coleta de dados. No total, 12 colaboradores, após aplicação dos critérios de inclusão e exclusão e aceite, compuseram a amostra.

Por sua vez, para a seleção dos acadêmicos, foram adotados como critérios de inclusão aqueles que já tinham realizado estágio curricular obrigatório em algum serviço de saúde e estivessem devidamente matriculados no quarto ano do curso de Enfermagem no ano de 2018, pois assim acreditava-se que já possuiriam um contato maior com as ações de promoção da saúde e prevenção de doenças desenvolvidas durante a graduação. Nenhum critério de exclusão foi estabelecido, resultando em 29 discentes elegíveis para a coleta de dados. Destes, 11 aceitaram participar voluntariamente da pesquisa.

A coleta de dados ocorreu por meio de entrevistas audiogravadas, com duração média de 15 minutos, as quais foram conduzidas por um questionário não estruturado com questões sociodemográficas e guiadas pelas seguintes questões norteadoras: para os profissionais: fale sobre as ações de promoção da saúde e prevenção de doenças que você elou sua equipe realiza; e para os acadêmicos: fale sobre sua experiência em ações de promoção da saúde e prevenção de doenças enquanto estagiário do curso de Enfermagem nos serviços de saúde.

Questões de apoio como "Qual sua percepção acerca da efetividade das ações desenvolvidas pela equipe?”, “Qual a sua percepção acerca da participação da comunidade nas ações desenvolvidas?” e "O que você entende por promoção da saúde e por prevenção de doenças?" também foram elaboradas para auxílio nas entrevistas. As mesmas foram realizadas até haver repetição das respostas e consequente saturação dos dados.

A coleta de dados com os acadêmicos ocorreu no mês de novembro de 2018, na instituição de ensino, em momentos nos quais os alunos compareciam ao campus e poderiam responder às questões sem comprometer seus afazeres. Os discentes eram abordados em sala de aula, havendo uma explicação quanto aos objetivos do estudo e princípios éticos de confidencialidade; em seguida, eles eram convidados para participar e encaminhados individualmente a uma sala reservada, caso tivessem interesse.

No que diz respeito aos profissionais de saúde, estes foram abordados no seu ambiente de trabalho, após agendamento prévio do pesquisador com a chefia da 
unidade. Caso aceitassem participar, eram encaminhados a uma sala reservada no local. A coleta de dados ocorreu no período de março a abril de 2019.

As entrevistas foram transcritas na íntegra e submetidas à análise de conteúdo, modalidade temática, seguindo-se as fases de pré-análise, exploração do material, tratamento dos resultados e interpretação. ${ }^{9}$ $\mathrm{Na}$ pré-análise, realizaram-se leituras flutuantes e exaustivas das falas para levantar os pontos relevantes perante os objetivos do estudo. $\mathrm{Na}$ fase de exploração do material ocorreu a codificação, processo pelo qual os dados brutos foram transformados sistematicamente e agregados em unidades. Na última fase, realizou-se a categorização, que consiste na classificação dos elementos segundo suas semelhanças e por diferenciação, com o posterior reagrupamento em função de características comuns, dando origem às categorias temáticas.

O estudo foi aprovado pelo Comitê de ética em Pesquisa com Seres Humanos da Universidade Estadual de Maringá, sob parecer número 3.910.456, no ano de 2018. Para este estudo, esclareceram-se a todos os participantes sobre a pesquisa e os preceitos éticos de acordo com a Resolução do Conselho Nacional de Saúde $n^{0} 466 / 2012$ e $n^{0} 510 / 2016$. Após lerem e concordarem em participar, eles assinavam o Termo de Consentimento Livre e
Esclarecido (TCLE) em duas vias de igual teor. Somente após esses procedimentos é que se dava início à entrevista.

Visando garantir o sigilo dos entrevistados, os servidores foram renomeados com a palavra "profissional", seguida pelo número arábico em ordem das entrevistas realizadas, por exemplo, "profissional 1", “profissional 2", "profissional 3" etc. O mesmo foi realizado com os discentes, sendo renomeados com a palavra "acadêmico", seguida de um número arábico, por exemplo, "acadêmico 1", “acadêmico 2" etc.

\section{RESULTADOS}

Quanto aos acadêmicos entrevistados, $90,9 \%$ eram do sexo feminino e a idade média foi de 24 anos ( \pm 2,5). Dentre os profissionais participantes, $91,7 \%$ eram do sexo feminino, tendo apresentado idade média de 43,5 anos $( \pm 11,6)$. Destes, $75 \%$ eram agentes comunitários de saúde, $16,7 \%$ técnicos de enfermagem e $8,3 \%$ auxiliares de enfermagem. Com relação à formação, $33,3 \%$ possuíam nível superior completo, $16,7 \%$ curso técnico, $41,7 \%$ ensino médio completo e $8,3 \%$ ensino médio incompleto.

As entrevistas, após analisadas, resultaram em duas categorias dispostas conforme a seguir. 


\section{A promoção da saúde e a prevenção de doenças}

Na primeira categoria, evidenciaramse as falas que expressavam o entendimento sobre os conceitos do tema da pesquisa e a contribuição que o curso de graduação forneceu para que pudessem compreender, teórica e tecnicamente, como se aplicam as atividades de promoção da saúde e prevenção de doenças, em especial mediante as estratégias empregadas na educação em saúde.

Por meio das falas, observou-se que o conceito de promoção da saúde envolvia o entendimento ampliado do mesmo, como preconizado pelo Ministério da Saúde:

Promoção a saúde seria a gente trabalhar o que a gente entende por saúde, não e só a ausência de doenças, mas é a gente valorizar e expor o que de fato é saúde, que é o bem-estar [...] passar esse conhecimento, o que é a saúde e de como ter saúde. (Acadêmico 9)

[...] é você promover uma melhoria na qualidade de vida, é você estar buscando métodos de estar promovendo e melhorando essa qualidade de vida. (Acadêmico 4)

[...] a gente está falando da qualidade de vida, de promover a saúde[...] que ele tenha desde o nascimento, seu desenvolvimento, até a velhice, uma vida não só livre da doença, mas que garantisse que ele passe bem por todas as fases da vida. (Profissional 5)

Entretanto, por vezes, relacionaram o conceito de promoção da saúde instantaneamente à prevenção de doenças:
[...] a gente aprende como promoção da saúde todas ações que promovam, que eduquem tanto a nossa equipe quanto o público, que eduquem para que não ocorra a incidência de novas doenças ou agravos de doenças crônicas... (acadêmico 1)

[...] estar mostrando para essa população essa promoção, uma melhor forma de estar prevenindo essas doenças. (Acadêmico 2)

Ter cuidado para não ficar doente. Tipo isso? Vacinar sempre que for preciso[...] Cuidar do quintal para não ficar doente, dengue essas coisas. (Profissional 3)

Prevenir as doenças... (Profissional 7)

Já quando questionados especificamente sobre a prevenção de doenças, ressaltaram a importância dessa estratégia para evitar a ocorrência de patologias, bem como complicações nos casos em que estas já estão instaladas:

São métodos e estratégias que você cria a partir de um problema que você encontra para você estar prevenindo que isso possa se agravar, então por exemplo se você tem uma doença crônica você tem que prevenir que outros pacientes tenham essa doença crônica[...] (Acadêmico 4)

É a gente trabalhar um ponto especifico do que a gente está tentando evitar, então a prevenção é trabalhar o que a gente não deseja, é trabalhar como que a gente pode evitar, os agravos ou as doenças. (Acadêmico 9)

Os acadêmicos destacaram a importância da graduação, em especial nas experiências de estágio, no preparo para o desenvolvimento de ações de promoção da saúde e prevenção de doenças:

A graduação ajudou bastante, que nem agora na unidade de saúde nos identificamos as 
necessidades daquela unidade, de acordo com as necessidades que fomos preparar as ações educativas, a gente via o que aquela população estava precisando e ai a gente ia montar de acordo para tentar diminuir[...] muitas coisas a gente pega na faculdade, muitas experiências daqui que a gente leva para lá [campo de estágio]. (Acadêmico 2)

Percebeu-se também que, por vezes, em decorrência do acúmulo de demanda pelos profissionais, as atividades desenvolvidas durante os estágios ajudaram no desenvolvimento do processo de trabalho:

Eu acredito que foram desenvolvidas muitas atividades, mas hoje ainda tem que ser mais devido ao curto tempo que eles (profissionais) têm para aplicar, então hoje essa relação faculdadeestagiário e o eSF consegue realizar mais promoções nessa área. (Acadêmico 9)

Durante o estágio eu presenciei algumas ações de promoção a saúde, acho que ainda existe um déficit muito grande em relação a isso, mas isso pode estar atrelado a grande demanda de atividades que o enfermeiro da atenção básica é responsável, então talvez seja por isso. (Acadêmico 10)

Ademais, durante as entrevistas, os discentes relataram uma fraqueza percebida ao vivenciar as práticas educativas nos campos de estágio. As estratégias adotadas pelas equipes de saúde se repetiam, sendo utilizados sempre os mesmos meios tradicionais para realizar as ações:

[...]eu senti bem defasada essa parte das ações educativas [...] distribuíram panfletos $e$ estava ótimo, e eles sempre chamam pessoas de fora para estar dando palestras. (Acadêmico 3)

Essa percepção foi reforçada pela fala dos profissionais:

Eu acredito que é o trabalho que a gente faz né, de casa em casa orientando o paciente para que ele não venha a ter um problema de saúde. (Profissional 1)

Saúde da família veio para prevenir as doenças, porque a gente teria que estar lá na casa prevenindo muitas coisas; diarreia, vomito, febre, se a mãe fizesse lá direitinho ele não precisava ir ao posto. (Profissional 7)[...]a gente promove a saúde. A gente faz palestras, a gente orienta. (Profissional 9)

\section{A realização das atividades de} promoção da saúde e prevenção de doenças na perspectiva dos profissionais

As orientações nas visitas domiciliares predominavam na educação da comunidade pela equipe. Entretanto, havia divergências quanto à percepção de adesão por parte da população: alguns profissionais acreditavam que as atividades são exitosas, enquanto outros as viam como pouco proveitosas e desvalorizadas pela comunidade:

Acho que são boas, tem um bom efeito na comunidade, tudo que é feito é valido. (Profissional 10)

Ótimo, quando a gente faz na minha área eu acho bem interessante, acho que eles realmente precisam disso. (Profissional 12) 
As vezes deixa muita coisa a desejar, não é assim uma realização total. (Profissional 3)

Acho que é muito fraca. Se tivesse mais incentivo, mais apoio. (Profissional 7)

Quando questionados sobre as facilidades e as dificuldades na realização das ações, identificaram-se os recursos humanos da equipe como fator facilitador da realização dessas atividades, enquanto os clientes, em sua maioria, dificultavam, não comparecendo e não aderindo às orientações. Já os recursos materiais fornecidos pela gestão da saúde, para alguns profissionais, não eram ofertados da maneira adequada, dificultando a realização de algumas ações idealizadas, enquanto para outros o estado se encontrava satisfatório e contribuía para a realização das atividades de promoção da saúde e de prevenção de doenças:

A maior dificuldade e a adesão do próprio cliente. As vezes a própria falta de consciência mesmo dele, é o que mais atrapalha, que as ações realmente tenham efeito. (Profissional 5)

As vezes a gente fala que vai ter teste rápido e não tem, as vezes vai fazer exame de diabetes e não tem aquela lâmina, então as vezes falta algumas coisas[...] Recursos. (Profissional 2)

A gente tem o apoio da equipe, sem isso a gente não conseguiria não. Somos em duas equipes aqui e uma ajuda a outra. (Profissional 11)
A gente tem bastante facilidade de conseguir materiais, a nossa chefe é bem empenhada. (Profissional 10)

\section{DISCUSSÃO}

Atualmente, a definiç̧ão de promoção da saúde que melhor expressa a ideia e subsidia as políticas públicas é orientada pela Carta de Ottawa, decorrente da Primeira Conferência Internacional sobre Promoção da Saúde, em novembro de 1986, no Canadá, que traz a seguinte caracterização: [...] o processo de capacitação da comunidade para atuar na melhoria de sua qualidade de vida e saúde, incluindo uma maior participação no controle deste processo [...] saúde é um conceito positivo, que enfatiza os recursos sociais e pessoais, bem como as capacidades físicas. ${ }^{10}$

O conceito de prevenção de doenças objetiva o não aparecimento de condições específicas e procede do conhecimento sobre a história natural da enfermidade para realizar intervenções específicas focadas em pontos relacionados às condições responsáveis pela incidência dessa patologia ou deterioração. ${ }^{11}$

As definições de promoção e prevenção devem ser trabalhadas desde a graduação, garantindo suas singularidades e consequente atuação fidedigna e efetiva. No caso dos cursos de graduação em 
Enfermagem em território nacional, os mesmos devem preparar os acadêmicos para a atuação profissional conforme os conceitos, princípios e diretrizes do SUS. ${ }^{4}$

Notou-se, no presente estudo, que houve diferenças na conceituação de promoção e prevenção por parte dos acadêmicos, o que demonstra uma fragilidade na formação dos mesmos em relação às demandas do $\mathrm{SUS}^{6}$. O apoio em uma educação humanista, crítica e reflexiva durante a graduação potencializa o desenvolvimento de atribuições que respondem às demandas do sistema de saúde. $^{12}$

Em contrapartida, um estudo realizado com coordenadores de cursos de graduação em Enfermagem identificou que a formação no Brasil prepara os acadêmicos para atuar em ações de promoção da saúde e prevenção de doenças, além de atividades gerenciais e técnicas, corroborando com o preconizado nas DCENF e nas demandas da $\mathrm{AB}$, respondendo, assim, de forma assertiva, às demandas populacionais. ${ }^{13}$

Uma pesquisa apresentada na Polônia, resumindo uma iniciativa piloto inovadora de alfabetização em saúde, sugere que os estudantes de saúde pública possam servir como educadores em saúde em atividades destinadas a desenvolver competências nessa área em indivíduos na atenção primária. Esse estudo observou que os profissionais de saúde estão bem posicionados para apoiar iniciativas de promoção da saúde e prevenção de doenças. ${ }^{14}$

Contexto similar ao estudo da Polônia ${ }^{14}$, nas falas dos entrevistados desta investigação, compreende-se que durante a vivência em UBS perceberam que as equipes possuem dificuldades na realização de ações de promoção e prevenção, de forma que a colaboração acadêmica se fazia indispensável. Ações de educação em saúde realizadas por equipes advindas das universidades beneficiam tanto a população envolvida quanto os acadêmicos, permitindo que os mesmos desenvolvam diversas competências. Ainda, a participação discente agrega valores construtivos para as ações já desenvolvidas nas unidades de saúde, confirmando as falas dos entrevistados. ${ }^{15}$

Nota-se a dificuldade na realização de ações de educação popular pela equipe de saúde, principalmente pelo excesso de afazeres, desvio de função e consequente falta de tempo. A burocratização do trabalho e o acúmulo de trabalho fazem os ACS não realizarem suas atribuições conforme preconizado na PNAB, o que pode acarretar consequências negativas nas ações voltadas à promoção da saúde. ${ }^{16}$

Consonando com os achados desta pesquisa, constata-se que ainda existe nas eSF características de um modelo 
tradicional de educação bancária, biomédico e reducionista, que por vezes pode levar à desvalorização do contexto biopsicossocial dos indivíduos e dos conhecimentos prévios dos mesmos. ${ }^{2,17} \mathrm{O}$ emprego de metodologias ativas no processo de aprendizagem para educação em saúde também não é comum às equipes, o que somado com os achados anteriores sugere um despreparo dos profissionais para a execução dessas atividades. $^{17}$

Uma revisão integrativa realizada com foco em ações educativas com hipertensos evidenciou que o uso de diferentes abordagens com os educandos pode proporcionar uma melhor compreensão e adesão às recomendações realizadas. ${ }^{18} \mathrm{O}$ emprego de metodologias ativas no processo educativo estimula uma transformação efetiva da realidade dos participantes, visto que são caracterizadas como processos interativos de educação, que buscam desenvolver a capacidade de observação e solução de questões nas quais o indivíduo está inserido, considerando os conhecimentos adquiridos e as experiências prévias, tornando-o autônomo e estimulando a ação-reflexão durante a interação com o profissional. ${ }^{12,17,18}$

As divergências nas opiniões referentes à percepção da população podem ser resultado da falta de um processo de avaliação da atividade. A análise crítica do processo educativo tem como intenção a busca de aperfeiçoamento das ações, assim como a reorientação e a recondução dos processos. Essa investigação acerca da percepção da população envolvida nas atividades é fundamental na interpretação dos resultados obtidos, situando educadores e educandos sobre as fragilidades e potencialidades das propostas educativas. As informações advindas das avaliações, primordialmente, podem subsidiar novas proposições pedagógicas/metodológicas. ${ }^{19}$

Um artigo realizado com os enfermeiros de ESF abordou a percepção que eles tinham sobre a realização de ações de educação para promoção da saúde. Os profissionais relataram, assim como no projeto de pesquisa em questão, a falta de colaboração da comunidade e a baixa disponibilidade de recursos materiais como fatores que dificultavam e desestimulavam a implementação das atividades no trabalho. ${ }^{20}$

A dicotomia nas opiniões com relação à visão da gestão como obstáculo ou facilitadora pode ser consequência das diferentes gestões locais. Mesmo com uma coordenação da Atenção Primária municipal, a equipe de saúde é gerenciada diretamente por um coordenador de cada unidade básica ${ }^{5}$, que guiará as atividades na área de abrangência conforme as necessidades de saúde da população e 
priorizações, que são influenciadas pelas características peculiares de cada região.

Para tanto, mostra-se importante que os acadêmicos e profissionais de saúde demonstrem constante aprendizado durante as vivências no contexto da saúde pública, seus princípios e diretrizes, uma vez que ainda há diversos cenários de aprendizagem quanto à complexidade do processo saúde-doença a serem explorados. Desse modo, observa-se a necessidade de manter o engajamento durante a gestão de trabalho e a identificação das necessidades de saúde e o cuidado centrado na pessoa de forma integrada.

\section{CONCLUSÃO}

Pode-se afirmar que, embora alguns acadêmicos e profissionais possuam uma visão ampliada do conceito de promoção da saúde e do seu desenvolvimento prático, outros ainda o confundem com a prevenção de doenças, o que pode gerar equívoco na prática profissional dos mesmos e das equipes nas quais estarão inseridos. Ademais, a atuação dos discentes enquanto estagiários nos serviços de saúde impulsiona e contribui para a realização dessas atividades, que, segundo os mesmos, não são comumente realizadas, principalmente devido à falta de tempo da equipe dos serviços.
Já quanto às facilidades e às dificuldades na realização de ações de educação em saúde visando à promoção do bem-estar da população, encontramos os recursos humanos da equipe como $\mathrm{o}$ principal fator facilitador na realização dessas atividades, enquanto os clientes, em sua maioria, dificultam, não comparecendo e não aderindo às recomendações. Por sua vez, os recursos materiais disponibilizados pela gestão da saúde, para alguns profissionais, possuem precariedade no seu fornecimento, enquanto para outros $\mathrm{O}$ estado atual está satisfatório e contribui para a realização das atividades de promoção da saúde e de prevenção de doenças no seu trabalho.

Observa-se como limitação a esta pesquisa a ausência da participação de servidores pertencentes às demais categorias profissionais e que atuam na AP, como médicos e enfermeiros. É imprescindível a realização de novos estudos com foco nessas populações para que, dessa forma, todo o contexto e perspectivas dos atores das equipes da AP sejam compreendidos e intervenções eficazes implementadas.

\section{REFERÊNCIAS}

1. Miranda GMD, Mendes ACGS, Silva ALA. Desafios das políticas públicas no cenário de transição demográfica e mudanças sociais no Brasil. Interface (Botucatu, Online) [Internet]. abr/jun 2017 
[citado em 22 jan 2020]; 21(61):309-20.

Disponível em:

https://www.scielo.br/j/icse/a/gtmDrRXmp

N3bbLDWRDCJcqw/?lang=pt

2. Malta DC, Morais NOL, Silva MMA,

Rocha D, Castro AM, Reis AAC, et al.

Política Nacional de Promoção da Saúde

(PNPS): capítulos de uma caminhada ainda

em construção. Ciênc Saúde

Colet. [Internet]. jun 2016 [citado em 22

jan 2020]; 21(6):1683-94. Disponível em:

https://www.scielo.br/j/csc/a/pWG9W7grq

FzzQGszmDKzvrb/?format=pdf\&lang=pt

3. Ministério da Saúde (Brasil). Portaria ${ }^{\circ}$

2.446, de 11 de novembro de 2014.

Redefine a Política Nacional de Promoção da Saúde (PNPS) [Internet]. Brasília, DF:

Ministério da Saúde; 2014. Disponível em: http://bvsms.saude.gov.br/bvs/saudelegis/g m/2014/prt2446_11_11_2014.html

4. Piovesan LR, Schimith MD, Simon BD, Budó MLD, Weiller TH, Bretas ACP. Promoção da saúde na perspectiva de enfermeiros de atenção básica. Esc. Anna Nery Rev Enferm. [Internet]. 2016 [citado em 17 dez 2021]; 24(3):e5816. Disponível em: https://www.e-

publicacoes.uerj.br/index.php/enfermagem uerj/article/view/5816/19396

5. Ministério da Saúde (Brasil). Portaria ${ }^{\circ}$ 2.436, de 21 de setembro de 2017. Aprova a Política Nacional de Atenção Básica, estabelecendo a revisão de diretrizes para a organização da Atenção Básica, no âmbito do Sistema Único de Saúde (SUS) [Internet]. Brasília, DF: Ministério da Saúde; 2017. Disponível em: http://bvsms.saude.gov.br/bvs/saudelegis/g m/2017/prt2436_22_09_2017.html 6. Conselho Nacional de Educação (Brasil), Câmara de Educação Superior. Resolução CNE/CES n ${ }^{\circ}$ 3, de 7 de novembro de 2001. Institui Diretrizes Curriculares Nacionais do Curso de Graduação em Enfermagem [Internet]. Brasília, DF: CNE; 2001. Disponível em: http://portal.mec.gov.br/cne/arquivos/pdf/C ES03.pdf

7. Carvalho VL, Oliveira ALC, Alves IKS, Silva RL, Silva CB. Competências para promoção da saúde em formandos dos cursos da área da saúde. Rev Enferm. UFPE. [Internet]. ago 2017 [citado em 24 fev 2020]; 11(Supl 8):3269-78. Disponível em:

https://periodicos.ufpe.br/revistas/revistaen fermagem/article/view/110193/22082

8. Figueiro MC, Amendoeira J. Intervenção contextualizada no domínio da promoção da saúde. Rev UIIPS [Internet]. 2018 [citado em 24 fev 2020]; 6(2):62-8.

Disponível em:

https://revistas.rcaap.pt/uiips/article/view/1 6131/14374

9- Bardin L. Análise de conteúdo. São

Paulo: Edições 70; 2011.

10.Carta de Ottawa. Primeira Conferência

Internacional de Promoção da Saúde.

[Internet]. Ottawa, nov 1986 [citado em 29 jun 2019]. Disponível em:

http://bvsms.saude.gov.br/bvs/publicacoes/ carta_ottawa.pdf

11. Czeresnia D. O conceito de saúde e a diferença entre prevenção e promoção. In: Czeresnia, D, Freitas CM. Promoção da saúde: conceitos, reflexões, tendências. 2 ed. Rio de Janeiro: Editora Fiocruz; 2009. p. 39-53.

12. Winters JRF, Prado ML, Heidemann ITSB. A formação em enfermagem orientada aos princípios do Sistema Único de Saúde: percepção dos formandos. Esc Anna Nery Rev Enferm. [Internet]. abr/jun 2016 [citado em 07 jun 2019]; 20(2):248-53. Disponível em: https://www.redalyc.org/pdf/1277/1277457 23006.pdf

13. Magnago C, Pierantoni CR. A formação de enfermeiros e sua aproximação com os pressupostos das Diretrizes Curriculares Nacionais e da Atenção Básica. Ciênc Saúde Colet. [Internet]. jan 2020 [citado em $24 \mathrm{fev}$ 2020]; 25(1):15-24. Disponível em: https://www.scielo.br/j/csc/a/QV8MBZ3Y qvMrPLXy9gNCV9w/?format=pdf\&lang= $\mathrm{pt}$

14. Janik-Koncewicz K, Rosik K, Młoźniak I, Zatoński M, Herbeć A, Zatoński W. Public health students as 
health educators: health awareness and behaviours among primary care professionals and public health students conducting a health literacy intervention. J Health Inequal. [Internet]. 2019 [citado em 20 mar 2020]; 5(1):89-95. Disponível em: https://www.termedia.pl/Public-healthstudents-as-health-educators-healthawareness-and-behaviours-amongprimary-care-professionals-and-publichealth-students-conducting-a-healthliteracy-intervention, 100,37641,1,1.html 15. Valente CA, Andrade V, Soares MBO, Silva SR. Atividades educativas no controle do câncer de colo do útero: relato de experiência. Rev Enferm Cent.-Oeste Min. [Internet]. set/dez 2015 [citado em 20 mar 2019]; 5(3):1898-904. Disponível em: http://www.seer.ufsj.edu.br/index.php/reco $\mathrm{m} /$ article/view/576/941

16. Machado LM, Pereira GS, Silva SO, Pieszak GM, Schimith MD, Rodrigues SO. Percepção dos agentes comunitários de saúde em relação à promoção da saúde nas visitas domiciliarias. Enferm Rev.

[Internet]. 2019 [citado em 25 fev 2020]; 22(1):47-55. Disponível em:

http://periodicos.pucminas.br/index.php/en fermagemrevista/article/view/20184 17.Lopes CR, Dalmolin IS, Durand MK, Rumor PCF, Heidemann ITSB, Koch C. Educação e cultura em saúde à luz de Paulo Freire. Rev Enferm UFPE on line
[Internet]. dez 2017 [citado em $25 \mathrm{fev}$ 2020]; 11(12):5122-8. Disponível em: https://periodicos.ufpe.br/revistas/revistaen fermagem/article/view/25338/25362 18. Vasconcelos MIO, Farias QLT, Nascimento FG, Cavalcante ASP, Mira QLM, Queiroz MVO. Educação em saúde na atenção básica: uma análise das ações com hipertensos. Rev APS [Internet]. abr/jun 2017 [citado 25 nov 2019]; 20(2):253-62. Disponível em:

https://periodicos.ufjf.br/index.php/aps/arti cle/view/15943/8283

19. Silva LAA, Schmidt SMA, Noal HC, Signor E, Gomes IEM. Avaliação da educação permanente no processo de trabalho em saúde. Trab Educ Saúde [Internet]. set/dez 2016 [citado $27 \mathrm{fev}$ 2020]; 14(3):765-81. Disponível em: https://www.scielo.br/j/tes/a/Lt5tHnB9CC DZCkP6hgxYCnS/?format=pdf\&lang=pt 20. Araújo WA, Assunção MLB, Araújo IS, Temoteo RCA, Souza EC, Almeida GS et al. Educação em saúde na Estratégia Saúde da Família: contribuições práticas do enfermeiro. Enferm Brasil [Internet]. 2018 [citado em 03 jan 2020]; 17(6):64553. Disponível em: https://portalatlanticaeditora.com.br/index. php/enfermagembrasil/article/view/2231/p df

RECEBIDO: 27/04/2020

APROVADO: 03/03/2021

PUBLICADO: dez/2021 